\title{
New low-mass pre-main sequence spectroscopic binaries in Orion ${ }^{\star}, \star \star$
}

\author{
E. Covino ${ }^{1}$, C. Melo ${ }^{2}$, J. M. Alcalá1 ${ }^{\text {, G. Torres }}{ }^{3}$, M. Fernández $^{4}$, A. Frasca ${ }^{5}$, and R. Paladino ${ }^{1}$ \\ 1 Osservatorio Astronomico di Capodimonte, Via Moiariello, 16, 80131 Napoli, Italy \\ 2 Observatoire de Genève, $51 \mathrm{ch}$. des Maillettes, 1290 Sauverny, Switzerland \\ 3 Harvard-Smithsonian Center for Astrophysics, 60 Garden Street, Cambridge MA 02138, USA \\ 4 Instituto de Astrofísica de Andalucía, Apdo. 3004, 18080 Granada, Spain \\ 5 Osservatorio Astrofisico di Catania, Via S. Sofia, 78, Città Universitaria, 95125, Catania, Italy
}

Received 19 February 2001 / Accepted 1 June 2001

\begin{abstract}
We report the results of a high-resolution spectroscopic monitoring campaign on low-mass pre-main sequence spectroscopic binaries, discovered recently in the general direction of the Orion star-forming region, based on ROSAT all-sky survey X-ray observations. Also included in the present study are two binaries recognized in the course of optical follow-up observations of X-ray sources in a selected sky strip crossing the Orion SFR perpendicular to the galactic plane. Orbital elements for the six double-lined spectroscopic binaries are derived from the analysis of the radial velocities of the components. The orbital periods span from 3 to 47 days. In addition, through a matching of the binary composite spectrum with synthetic binary spectra, we estimate spectral types and luminosity ratios for the components and derive lithium abundances for individual binary components. Using the estimated stellar parameters combined with kinematical information and lithium abundance determinations, we examine the evolutionary status of the objects. We then use the minimum masses derived from the solution of the spectroscopic orbits for the systems of confirmed PMS nature to make comparisons with current theoretical pre-main sequence evolutionary tracks, and attempt to set constraints on some of the most frequently used models.
\end{abstract}

Key words. stars: binaries: spectroscopic - stars: pre-main sequence - stars: low-mass, brown dwarfs stars: fundamental parameters

\section{Introduction}

Binary stars are at the basis of our knowledge of fundamental astrophysical parameters such as stellar masses and radii. However, for pre-main sequence (PMS) stars, estimates of the stellar mass typically rely on rather uncertain comparison with theoretical evolutionary tracks on the HR diagram. Therefore, the dynamical determination of stellar masses is strongly needed in order to obtain reliable estimates of stellar ages as well as to test current theories of early stellar evolution.

\footnotetext{
Send offprint requests to: E. Covino,

e-mail: covino@na.astro.it

* Based on observations with the Calar Alto Observatory $2.2 \mathrm{~m}$ telescope, the Swiss Euler telescope (La Silla-Chile), and the Multiple Mirror Telescope, a joint facility of the Smithsonian Institution and the University of Arizona.

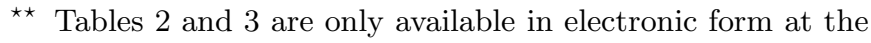
CDS via anonymous ftp to

cdsarc.u-strasbg.fr $(130.79 .128 .5)$ or via

http://cdsweb.u-strasbg.fr/cgi-bin/qcat?J/A+A/375/130
}

Among all kinds of binaries, spectroscopic systems, allowing one to probe the short period regime (or, equivalently, the small separation domain), have a particularly important role for the understanding of the formation and early evolution of stars. Yet, in spite of their importance, until very recently only a handful of PMS spectroscopic binaries (SB) have been known and analysed in the literature (see Mathieu 1999 for a recent review).

Moreover, as suggested by Mayor \& Mermilliod (1984), the dynamical evolution of short-period binaries with lowmass components depends mainly on their PMS history. Therefore, the study of orbital parameters for a statistically significant sample of PMS binaries is necessary in order to gain insight into the formation process and early evolution of close binary systems, as well as to provide clues on the time scales for tidal circularization of the orbits and spin-orbit synchronization processes (Melo et al. 2001).

These and other important issues in early stellar evolution can be addressed by studying PMS binary systems. 
In particular, the study of i) binary frequency and distribution of orbital periods, ii) distributions of masses and mass ratios of the components, and iii) orbital eccentricities versus orbital periods will allow us to learn more about the formation mechanisms of close binaries relative to the initial angular momentum of the protostellar cloud, and understand how the existence and life-time of circumstellar/circumbinary disks can be affected by the presence of close companions, and to what extent the occurrence and evolution of such disks can affect the orbit-spin angular momentum exchange, and the distribution of orbital eccentricities.

In order to address all these questions, the statistics on pre-main sequence SB systems need to be enlarged significantly. In particular, in order to derive information on stellar masses of the components as well as on other orbital parameters, it is necessary first of all to obtain a solution of the binary orbits.

In the present paper we report the results of a highresolution spectroscopic monitoring campaign on PMS double-lined spectroscopic binary (SB2) systems in the Orion star-forming region. These objects were identified among the optical counterparts of ROSAT X-ray sources in the Orion star formation region (SFR) and classified as weak-T Tauri star (WTTS) candidates on the basis of medium/low resolution spectroscopy (Alcalá et al. 1996, hereafter, Paper I). Subsequent high-resolution spectroscopic observations of the Orion WTTS candidates have allowed us to confirm the PMS nature for most of these objects, as well as to discover among them a number of double-line spectroscopic binaries (Alcalá et al. 2000, hereafter, Paper II). Also included in the present study are two spectroscopic binaries recognized in the course of optical follow-up observations of X-ray sources in a selected strip of sky crossing the Orion SFR perpendicular to the galactic plane (Alcalá et al. in preparation). Besides the spectroscopic monitoring, a parallel photometric campaign has been undertaken by us with the aim to search for and then study possible eclipsing systems among our SB sample. In fact, the discovery of the first low-mass PMS, spectroscopic and eclipsing binary has been recently reported (Covino et al. 2000).

The layout of the paper is the following. In Sect. 2, we present the observations and describe the data reduction. In Sect. 3, where the bulk of the results are presented, we introduce the methods applied for the data analysis and the determination of radial and rotational velocities and report the results obtained from the analysis of the radial velocity curves and the solution of the spectroscopic orbits. Then we present the main criteria used to discriminate low-mass PMS stars from eventual more evolved objects and describe i) the method used for estimating the spectral type and the fractional flux contributed by each individual component to the combined flux; ii) the determination of Li I $6708 \AA$ line equivalent widths and the derivation of lithium abundance for each binary component; iii) examine the spatial location and kinematics of the objects, and iv) locate the individual components of four of the investigated systems on the HR diagram. In Sect. 4 , we provide some clues to the evolutionary status of the individual systems, and in Sect. 5 the comparison is made with different sets of theoretical PMS evolutionary tracks. The results are finally summarized and discussed in Sect. 6.

\section{Spectroscopic observations and data reduction}

The high-resolution spectroscopic observations were conducted in multiple runs using a number of different telescopes. The observing protocols and reduction procedures are somewhat different for each telescope, as described below. Observations with the Fiber Optics Cassegrain Echelle Spectrograph (FOCES) were carried out at the $2.2 \mathrm{~m}$ telescope at the Calar Alto Observatory in Spain, in 1997, 1998, and 1999, as detailed in Table 1.

Some seventy echelle orders are included in these spectra, covering a range from 3900 to $7000 \AA$, with a nominal resolving power $\lambda / \Delta \lambda$ of about 30000 , using a 2 arcsec aperture on the sky. The reduction of the FOCES spectra was performed using IDL routines specifically written for that instrument (Pfeiffer et al. 1998). More details on the reduction of FOCES data can be found in Paper II.

High-resolution echelle spectra were also taken with the two-fiber-fed high-resolution spectrograph CORALIE mounted on the $1.2 \mathrm{~m}$ Swiss Euler telescope, at La Silla, Chile. CORALIE is an upgraded version, although very similar, of the spectrograph ELODIE mounted on the $1.93 \mathrm{~m}$ telescope at Haute de Provence Observatory which is described in Baranne et al. (1996), while Queloz et al. (2000) briefly discuss some differences between the two instruments. The CORALIE spectra, covering a wavelength range from 3690 to $6900 \AA$ with a resolving power of 47000 , were collected during two observing runs in December 1998 and January 1999. All observations were taken with one fiber centered on the target star and the other fiber illuminated by the background sky. During non-dark time, the sky fiber information is important in order to clean the stellar spectrum of eventual Moon light contamination. The reduction is performed by an on-line reduction procedure: after reading the CCD, the spectrum is extracted, calibrated in wavelength and flatfielded. The on-line reduction system also performs the cross-correlation of the stellar spectrum with a numerical mask (Queloz 1995) for the determination of radial and rotational velocities. Details are given in the next section.

Additionally, high-resolution single-order echelle spectra were obtained at the Harvard-Smithsonian Center for Astrophysics (CfA) using the Multiple Mirror Telescope (MMT) in Arizona, the $1.5 \mathrm{~m}$ Tillinghast reflector at the Fred L. Whipple Observatory (FLWO), also in Arizona, and the $1.5 \mathrm{~m}$ Wyeth reflector at the Oak Ridge Observatory (ORO), in Massachusetts. All three telescopes were used during multiple runs between 1996 and 1999. The observations have a resolving power of $\lambda / \Delta \lambda \approx 35000$, and cover a $45 \AA$ wavelength range 
Table 1. Journal of observations.

\begin{tabular}{|c|c|c|c|c|}
\hline $\begin{array}{c}\text { Observing } \\
\text { period }\end{array}$ & telescope & instrument & $\begin{array}{c}\lambda \text { range } \\
{[\AA]}\end{array}$ & $\begin{array}{c}\text { Resolution } \\
\lambda / \Delta \lambda\end{array}$ \\
\hline 1997 Oct. 09-15 & $\mathrm{CA} 2.2 \mathrm{~m}$ & FOCES & $4500-8000$ & 30000 \\
\hline 1997 Dec. 08-16 & $"$ & $"$ & $"$ & $"$ \\
\hline 1998 Aug. 05-09 & $"$ & $"$ & $"$ & $"$ \\
\hline 1998 Sep. 01-09 & $"$ & $"$ & $"$ & $"$ \\
\hline 1998 Dec. $21-25$ & $"$ & $"$ & $"$ & $"$ \\
\hline 1999 Jan. 03-07 & $"$ & $"$ & $"$ & $"$ \\
\hline 1998 Dec. 07-20 & LS 1.2 m Euler & CORALIE & $3690-6900$ & 47000 \\
\hline 1999 Jan. 22-Feb. 06 & $"$ & $"$ & $"$ & $"$ \\
\hline 1996-1999 & CfA MMT & sing.-ord. ech. & $5165-5211$ & 35000 \\
\hline 1996-1999 & CfA $1.5 \mathrm{~m}$ FLWO & $"$ & $"$ & $"$ \\
\hline 1996-1999 & CfA $1.5 \mathrm{~m}$ ORO & $"$ & $"$ & $"$ \\
\hline
\end{tabular}

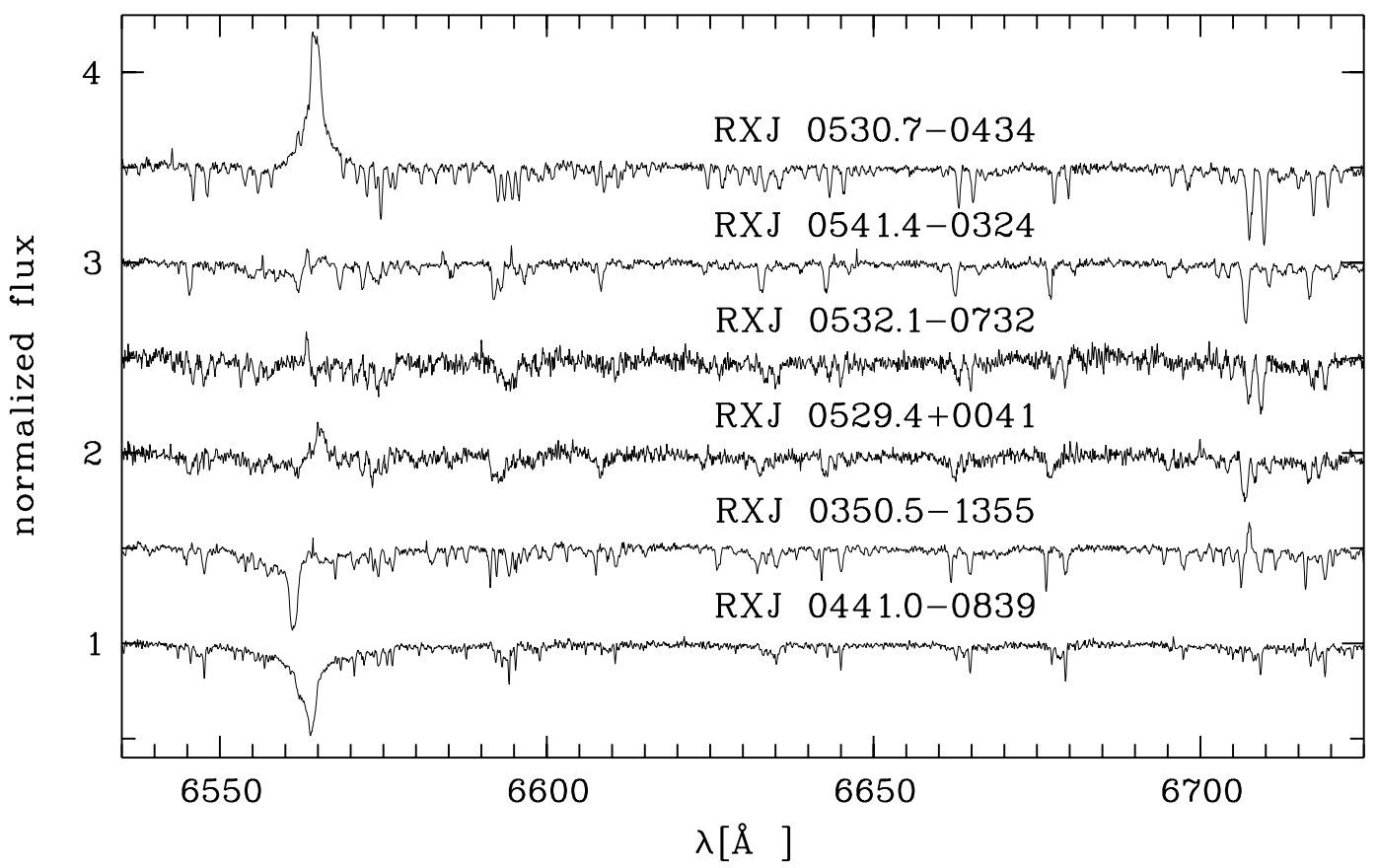

Fig. 1. Examples of FOCES spectra for the six double-lined spectroscopic binaries of our sample, in the wavelength range spanning from $\mathrm{H} \alpha$ to the $\mathrm{Li}$ I $\lambda 6708 \AA$ and $\mathrm{Ca}$ I $\lambda 6718 \AA$ lines. The third component is clearly distinguishable in the spectra of RX J0441.0-0839 and RX J0529.4+0041.

centered at $5187 \AA$. The zero point of the radial velocity scale for observations at the three telescopes was set by monitoring the sky during all runs with multiple dusk and dawn exposures. The residual velocities (after heliocentric correction) measured from the sky spectra, which should be zero under ideal conditions, were averaged over each run and applied as corrections to the stellar measurements. Thus, by construction, observations at the three CfA telescopes are on the same system. The reduction of these spectra was carried out as described in Neuhäuser et al. (1995).

The journal of observations is summarised in Table 1. Examples of spectra of the 6 binaries studied here are presented in Fig. 1.

\section{Results}

\subsection{Radial and rotational velocity determinations}

We derived radial velocities (RV) and projected rotational velocities, $v \sin i$, by using cross-correlation techniques, using the same procedure described in Covino et al. (1997).

The details concerning the measurements of RV and $v \sin i$ from FOCES spectra are described in Paper II. The estimated mean errors are below $1 \mathrm{~km} \mathrm{~s}^{-1}$ for RV, and of about $10 \%$ for $v \sin i$.

The CORALIE on-line reduction system performs the cross-correlation of the stellar spectrum with a K0V CORAVEL-type numerical mask (Queloz 1995) giving as a result a cross-correlation function (CCF). The CCF is then fitted by a Gaussian 
$g(V)=1-D \exp \left(-\left(V-V_{\mathrm{r}}\right)^{2} / 2 \sigma_{\mathrm{CCF}}^{2}\right)$. The fit gives us: (1) the stellar radial velocity, $V_{\mathrm{r}}$, which is the center of the CCF, (2) the CCF depth $D$, and (3) the CCF width, $\left(\sigma_{\mathrm{CCF}}\right)$, which is related to line broadening mechanisms, such as stellar rotation. When the projected stellar rotational velocity $v \sin i$ is greater than about $30 \mathrm{~km} \mathrm{~s}^{-1}$ the CCF cannot be fitted by a Gaussian profile anylonger, and, in these cases, another fit function is used, which is the result of the convolution of the CCF of a slowly rotating $\mathrm{K} 2 \mathrm{~V}$ star with the rotational profile evaluated at a given $v \sin i$ (Gray 1976). A least-square fit is then performed to the observed CCF. As in the Gaussian fits described before, this procedure gives the radial velocity, the depth $\mathrm{D}$ of the $\mathrm{CCF}$, and the $v \sin i$ of the observed star.

For CORALIE the radial-velocity precision can be written (Barrane et al. 1996, Eq. (9)) as:

$\epsilon_{\mathrm{p}}\left(V_{\mathrm{r}}\right)=\frac{K}{D S / N} \frac{(1+0.2 w)}{3}\left(\mathrm{~km} \mathrm{~s}^{-1}\right)$

where $w$ is $F W H M$ of the observed CCF, D its relative depth, $S / N$ the signal-to-noise in the CCF continuum. $K$ is a constant which depends on the correlation template used and the spectral type of the observed star. For our program stars, $K$ is equal to $0.03 \mathrm{~km} \mathrm{~s}^{-1}$ (Queloz 1995). The intrinsic broadening of the cross-correlation function of a non-rotating star as a function of $(B-V)$ is a 2 nd order effect which is only important for very slow rotators, with $v \sin i$ smaller than about $3 \mathrm{~km} \mathrm{~s}^{-1}$ (Queloz 1998). Nonetheless, the aim of the paper is not to yield very precise rotation (better than $1 \mathrm{~km} \mathrm{~s}^{-1}$ ) and, among the stars presented in this paper, only the slowly rotating components of RX J0441.0-0839 may be affected by this problem. The typical values of $S / N, D$ and $w$ for our program stars give an internal error on the radial-velocity measurement of about $100 \mathrm{~m} \mathrm{~s}^{-1}$.

Radial velocities from the CfA spectra were obtained using TODCOR (Zucker \& Mazeh 1994), a twodimensional cross-correlation technique that uses a combination of two templates, one for each component of the binary. The two-dimensional correlation function is expressed as a combination of standard one-dimensional correlation functions computed using the $\mathrm{IRAF}^{1}$ task XCSAO (Kurtz \& Mink 1998). The templates were chosen from an extensive grid of synthetic spectra based on model atmospheres by Kurucz (1992a, 1992b), available for a range of effective temperatures, projected rotational velocities, surface gravities, and metallicities. TODCOR is well suited to the relatively low signal-to-noise ratios of these spectra, and has the advantage of greatly reducing the systematic errors in the velocities that occur in traditional methods when the two peaks in the (one-dimensional) correlation function are blended (see also Latham et al. 1996).

${ }^{1}$ IRAF is distributed by the National Optical Astronomy Observatories, which is operated by the Association of Universities for Research in Astronomy, Inc., under contract with the National Science Foundation.
The formal uncertainties in the individual velocities we derive are between 0.5 and $3.0 \mathrm{~km} \mathrm{~s}^{-1}$, depending on the rotational velocity and the signal-to-noise ratio. Three of our targets turn out to be triple-lined. In this case, we used an extension of TODCOR to three dimensions (Zucker et al. 1995).

A large number of correlations were performed using TODCOR in order to determine the best template for each component of each binary. In addition to the radial velocities, we determined the rotational broadening by correlating against templates with a range of $v \sin i$ values, and then interpolating in a table of correlation value versus $v \sin i$ to maximize the average correlation over all exposures of the object. The errors in the rotational velocities from the CfA spectra are estimated to be between 2 and $3 \mathrm{~km} \mathrm{~s}^{-1}$.

The individual radial velocity measurements as a function of heliocentric Julian Day are reported for all the systems in Table $2^{2}$. In three cases we detected a third component in the system, and were able to measure its velocity with either CORALIE or the CfA telescopes. These measurements are given separately in Table $3^{2}$.

\subsection{Solution of spectroscopic orbits}

Spectroscopic orbital solutions for each of our targets were derived using standard non-linear least squares techniques (e.g., Press et al. 1992). The orbital elements are the period, $P$, the radial velocity of the center of mass, $\gamma$, the semi-amplitudes of the radial velocity curves of each component, $K_{1}$ and $K_{2}$, the eccentricity, $e$, the longitude of periastron, $\omega$, and the time of periastron passage, $T$. For circular orbits, the epoch $T$ corresponds to the first quadrature (i.e., most massive component receding at maximum radial velocity). Derived quantities include the mass ratio, $q$, the minimum masses of the components, $M \sin ^{3} i$, and the projected semi-major axes, $a \sin i$.

Because of the variety of telescopes and instruments used to measure the radial velocities of our six targets, some care is required in order to account for possible systematic differences between CORALIE, FOCES, and CfA observations, as well as differences in the internal precision. In particular, we have allowed for differences in the zero point of the velocity scale by including an offset for the FOCES and CfA velocities, and adopting the CORALIE system as the reference because of the higher intrinsic precision of those observations. These offsets were included as unknowns in the least-squares problem, and are solved for simultaneously with the other orbital elements.

In addition, the relative weights of observations from the three telescopes were determined by re-normalizing the internal errors of each set of observations to the standard deviation of the corresponding residuals from a preliminary solution, while maintaining the relative weights of the individual velocities within each series. This procedure was iterated until convergence.

\footnotetext{
${ }^{2}$ Available only in electronic form at the CDS.
} 

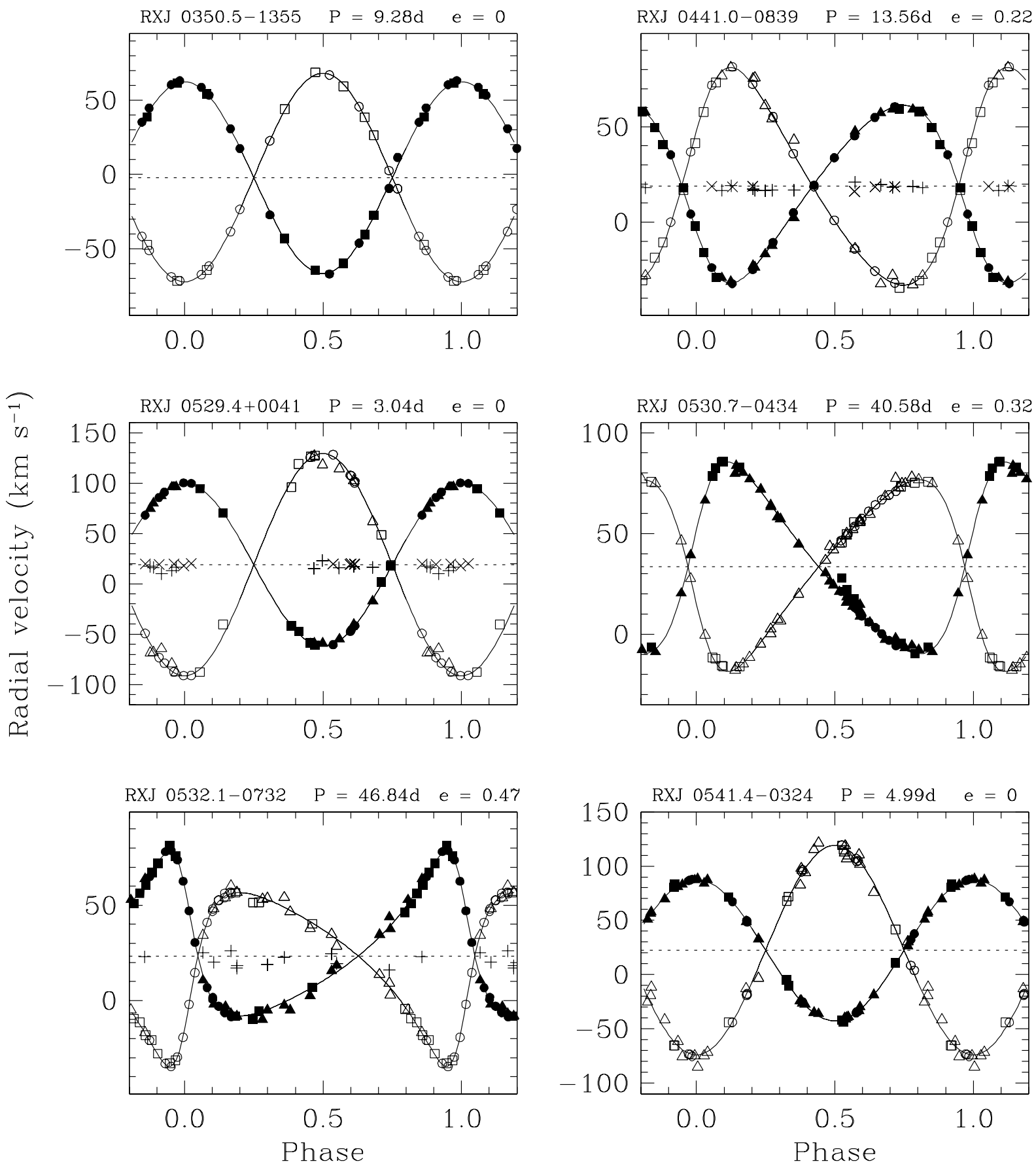

Fig. 2. Radial velocity curves for the six double-lined spectroscopic binaries. The data points for the primary and secondary components are represented by filled and open symbols, respectively (circles for CORALIE, squares for FOCES, and triangles for CfA observations); the orbital fits are drawn as solid lines and the dotted lines indicate the center-of-mass velocity. Velocities for the third components in three of our systems are represented with crosses (CORALIE) and plus signs (CfA).

As a test, separate solutions were derived for each telescope when allowed by the number of observations. No significant differences in the elements were seen other than trivial offsets in the $\gamma$ velocity. Therefore, for the final solutions we combined the observations as described above. The orbital elements and derived quantities from the fits are listed in Table 4. These fits are shown together with the observations in Fig. 2. In three cases the formal ec- centricity turned out to be insignificant, and we therefore adopted circular orbits. Three of the systems are triplelined, and the velocities measured for the third component are also plotted and are near the center-of-mass velocity of the binary in all cases. In the case of the eclipsing system RX J0529.4+0041 the orbital period is derived from the photometry (Covino et al. 2000). 
Table 4. Orbital parameters from spectroscopic orbital solutions.

RX J0350.5-1355 RX J0441.0-0839 RX J0529.4+0041 e RX J0530.7-0434 RX J0532.1-0732 RX J0541.4-0324

\begin{tabular}{|lcccccc|}
\hline \hline & & & & & \\
$P_{\text {orb }}[\mathrm{d}]$ & $9.2790 \pm 0.0036$ & $13.5601 \pm 0.0012$ & $3.03772 \pm 0.00001$ & $40.5738 \pm 0.0047$ & $46.850 \pm 0.011$ & $4.989169 \pm 0.000067$ \\
$T(\mathrm{HJD}-2400000)^{a}$ & $51167.0132 \pm 0.0034$ & $51321.583 \pm 0.027$ & $51336.1194 \pm 0.0013$ & $51098.944 \pm 0.073$ & $51207.754 \pm 0.027$ & $51155.9236 \pm 0.0014$ \\
$e$ & 0 & $0.2156 \pm 0.0022$ & 0 & $0.3186 \pm 0.0020$ & $0.4726 \pm 0.0024$ & 0 \\
$\gamma\left(\mathrm{km} \mathrm{s}^{-1}\right)$ & $-2.12 \pm 0.14$ & $+18.853 \pm 0.084$ & $+18.99 \pm 0.14$ & $+33.44 \pm 0.11$ & $+23.39 \pm 0.12$ & $+22.559 \pm 0.081$ \\
$\omega(\mathrm{deg})$ & 0 & $114.51 \pm 0.69$ & 0 & $286.91 \pm 0.56$ & $55.11 \pm 0.31$ & 0 \\
$K_{1}\left(\mathrm{~km} \mathrm{~s}^{-1}\right)$ & $64.58 \pm 0.29$ & $46.61 \pm 0.15$ & $80.58 \pm 0.15$ & $47.51 \pm 0.21$ & $43.25 \pm 0.18$ & $65.16 \pm 0.10$ \\
$K_{2}\left(\mathrm{~km} \mathrm{~s}^{-1}\right)$ & $70.20 \pm 0.18$ & $57.13 \pm 0.28$ & $110.31 \pm 0.30$ & $47.59 \pm 0.15$ & $45.61 \pm 0.15$ & $96.63 \pm 0.56$ \\
$a_{1} \sin i(\mathrm{Gm})$ & $8.241 \pm 0.039$ & $8.486 \pm 0.030$ & $3.3661 \pm 0.0067$ & $25.13 \pm 0.11$ & $24.56 \pm 0.11$ & $4.4700 \pm 0.0074$ \\
$a_{2} \sin i(\mathrm{Gm})$ & $8.957 \pm 0.024$ & $10.402 \pm 0.055$ & $4.608 \pm 0.013$ & $25.168 \pm 0.083$ & $25.90 \pm 0.10$ & $6.629 \pm 0.040$ \\
$q$ & $0.9200 \pm 0.0051$ & $0.8158 \pm 0.0052$ & $0.7305 \pm 0.0025$ & $0.9984 \pm 0.0049$ & $0.9483 \pm 0.0056$ & $0.6743 \pm 0.0042$ \\
$M_{1} \sin ^{3} i\left(M_{\odot}\right)$ & $1.2259 \pm 0.0090$ & $0.8041 \pm 0.0092$ & $1.2652 \pm 0.0080$ & $1.541 \pm 0.014$ & $1.197 \pm 0.011$ & $1.307 \pm 0.017$ \\
$M_{2} \sin ^{3} i\left(M_{\odot}\right)$ & $1.128 \pm 0.011$ & $0.6560 \pm 0.0057$ & $0.9242 \pm 0.0045$ & $1.538 \pm 0.016$ & $1.135 \pm 0.012$ & $0.8815 \pm 0.0069$ \\
No. of meas. & $13 / 8 / 0$ & $12 / 8 / 12$ & $12 / 8 / 10$ & $10 / 11 / 32$ & $16 / 12 / 18$ & $15 / 8 / 26$ \\
$r m s_{1}\left(\mathrm{~km} \mathrm{~s}^{-1}\right)$ & $2.22 / 0.97 /-$ & $0.30 / 2.10 / 1.39$ & $0.62 / 1.24 / 1.72$ & $0.49 / 3.28 / 1.78$ & $0.68 / 1.72 / 2.95$ & $0.33 / 1.90 / 1.31$ \\
$r m s_{2}\left(\mathrm{~km} \mathrm{~s}^{-1}\right)$ & $0.49 / 1.08 /-$ & $0.59 / 3.11 / 3.75$ & $1.08 / 5.87 / 7.58$ & $0.43 / 1.39 / 1.57$ & $0.65 / 1.99 / 2.38$ & $1.76 / 2.83 / 7.83$ \\
$\left.\Delta R V_{\text {FOCES }}(\mathrm{km} \mathrm{s})^{-1}\right)^{c}$ & $-1.33 \pm 0.22$ & $+0.57 \pm 0.48$ & $+0.04 \pm 0.26$ & $-1.19 \pm 0.16$ & $+0.92 \pm 0.19$ & $-1.02 \pm 0.51$ \\
$\Delta R V_{\text {CfA }}\left(\mathrm{km} \mathrm{s}^{-1}\right)^{d}$ & - & $+1.92 \pm 0.41$ & $+3.53 \pm 0.55$ & $+0.66 \pm 0.23$ & $-0.36 \pm 0.46$ & $+1.12 \pm 0.27$ \\
Time span (days) & 30.7 & 714.2 & 1064.1 & 1445.0 & 1064.1 & 1092.0 \\
\hline
\end{tabular}

Notes to Table 4:

a) For eccentric orbits this is the time of periastron passage, and for circular orbits it is the time of maximum primary velocity.

b) Number of measurements used for the orbital solution from CORALIE, FOCES and CfA, respectively.

c) Radial velocity shift between FOCES and CORALIE observations.

d) Radial velocity shift between CfA and CORALIE observations.

e) Orbital period derived from photometric light-curve solution (Covino et al. 2000).

Table 5. Derived astrophysical parameters of the binary components.

\begin{tabular}{|c|c|c|c|c|c|c|}
\hline & RX J0350.5-1355 & RX J0441.0-0839 & RX J0529.4+0041 ${ }^{a}$ & RX J0530.7-0434 & RX J0532.1-0732 & RX J0541.4-0324 \\
\hline $\mathrm{SpT}_{1}$ & $\mathrm{~K} 0-\mathrm{K} 1$ & G3 & $\mathrm{K} 1-\mathrm{K} 2$ & $\mathrm{~K} 2-\mathrm{K} 3$ & $\mathrm{~K} 2-\mathrm{K} 3$ & G8 \\
\hline $\mathrm{SpT}_{2}$ & $\mathrm{~K} 1-\mathrm{K} 2$ & G8 & K7-M0 & K2-K3 & K3 & K3 \\
\hline$T_{\text {eff }, 1}$ & $4900 \pm 200$ & $5500 \pm 200$ & $5025 \pm 100$ & $4600 \pm 200$ & $4600 \pm 200$ & $5100 \pm 200$ \\
\hline$T_{\text {eff }, 2}$ & $4800 \pm 200$ & $5100 \pm 200$ & $4020 \pm 200$ & $4600 \pm 200$ & $4500 \pm 200$ & $4500 \pm 200$ \\
\hline$l_{2} / l_{1}$ & $0.82 \pm 0.05$ & $0.70 \pm 0.05$ & $0.25 \pm 0.05$ & $1.00 \pm 0.05$ & $0.82 \pm 0.05$ & $0.25 \pm 0.05$ \\
\hline$L_{1}\left(L_{\odot}\right)$ & - & - & $+1.75 \pm 0.15$ & $5.35 \pm 0.25$ & $1.53 \pm 0.05$ & $4.70 \pm 0.1$ \\
\hline$L_{2}\left(L_{\odot}\right)$ & - & - & $+0.35 \pm 0.15$ & $5.35 \pm 0.25$ & $1.47 \pm 0.05$ & $1.20 \pm 0.1$ \\
\hline$v_{1} \sin i\left(\mathrm{~km} \mathrm{~s}^{-1}\right)$ & $19 \pm 2$ & $2 \pm 1$ & $25 \pm 3$ & $13 \pm 2$ & $20 \pm 2$ & $22 \pm 2$ \\
\hline$v_{2} \sin i\left(\mathrm{~km} \mathrm{~s}^{-1}\right)$ & $7 \pm 1$ & $1 \pm 1$ & $17 \pm 2$ & $13 \pm 2$ & $22 \pm 2$ & $15 \pm 2$ \\
\hline$W_{1}(\mathrm{Li})(\mathrm{m} \AA)$ & $210 \pm 20$ & $200 \pm 20$ & $370 \pm 30$ & $580 \pm 30$ & $490 \pm 20$ & $320 \pm 20$ \\
\hline $\begin{array}{l}W_{2}(\mathrm{Li})(\mathrm{m} \AA) \\
\text { corrected }\end{array}$ & $250 \pm 20$ & $135 \pm 20$ & $450 \pm 50$ & $580 \pm 30$ & $470 \pm 20$ & $400 \pm 50$ \\
\hline $\log N(\mathrm{Li})_{1}$ & $2.6 \pm 0.2$ & $3.1 \pm 0.3$ & $3.2 \pm 0.3$ & $3.3 \pm 0.2$ & $3.2 \pm 0.3$ & $3.1 \pm 0.2$ \\
\hline $\log N(\mathrm{Li})_{2}$ & $2.7 \pm 0.2$ & $2.5 \pm 0.3$ & $2.4 \pm 0.5$ & $3.2 \pm 0.2$ & $3.1 \pm 0.3$ & $2.9 \pm 0.3$ \\
\hline
\end{tabular}

Note to Table 5:

$a$ : Stellar parameters derived from combined solution of radial velocity and light curves.

\subsection{Spectral types and relative fluxes of binary components}

Spectral types and relative fluxes for the individual components of the spectroscopic binaries in our sample have been obtained from the FOCES observations through a matching of the observed binary spectrum with that of a synthetic binary. None of the studied systems contains classical T Tauri stars, as indicated by the weak emission characteristics observed in their spectra, thus no spectral veiling is expected to affect the photospheric lines.

Relative fluxes for the individual SB2 components have been first estimated from measurements of the intensities 
of individual peaks in the CCF with respect to the total one, following the prescription by Lee et al. (1994). If the spectral types of the binary components are not very different (within one spectral class), the relative intensities of the CCF peaks provide a good proxy of individual stellar fluxes to the combined flux. Synthetic binary spectra have then been constructed by combining the spectra of slowly rotating standard stars, of known spectral type, properly shifted in radial velocity, scaled by appropriate factors, and convolved with a suitable rotation profile, to account for rotational broadening. The spectral types of the components are then estimated through a matching of the binary spectrum with the synthetic one. The observed spectra used for this purpose are chosen among those with better signal to noise ratio, in which the components are seen at the largest separation. An example of the result of this procedure for deriving the spectral type and flux ratio of the individual binary components is shown in Fig. 3.

The spectral types and flux ratios for the individual components are reported in Table 5. The estimated uncertainty on the spectral type of each component is estimated to be one spectral subclass, but it can be of two subclasses in the worst case. The uncertainty on the flux ratio of individual components is within $10 \%$. Effective temperatures have finally been derived from the spectral types adopting the temperature scale given by de Jager \& Nieuwenhuijzen (1987), for luminosity class IV.

\subsection{Lithium abundance determination}

Critical to this study is to find confirmation that the objects in our sample are bona fide PMS stars.

The presence of a strong Li I $\lambda 6708 \AA$ absorption line is regarded as the primary indicator of stellar youth and is therefore used as the most important criterion to recognize low-mass PMS stars. FOCES spectra, as those shown in Fig. 1, in which the binary components are seen at the largest radial velocity separation are used to derive $\mathrm{Li}$ equivalent widths, $W(\mathrm{Li})$, for the individual stars. However, in the case of spectroscopic binaries (or multiples), the observed equivalent widths are measured relatively to the total stellar continuum and have therefore to be corrected for the "binarity" effect, i.e. they need to be scaled by an appropriate correction factor which corresponds to the actual contribution of each component to the total observed continuum. The correction factor to be applied to the measured equivalent width of each component has been evaluated from the ratio of the intensities of the peaks in the cross-correlation function between the binary spectrum and the spectrum of a standard star of similar spectral type, as described in Sect. 3.3. Analogous treatment was followed in the cases where a third component is present, but performing a three-components fit.

Equivalent widths of the Li I 6708 line have been measured by two-components (three-components in the case of spectroscopic triples) Gaussian fits on the residual spectrum obtained by subtracting the synthetic binary spec-

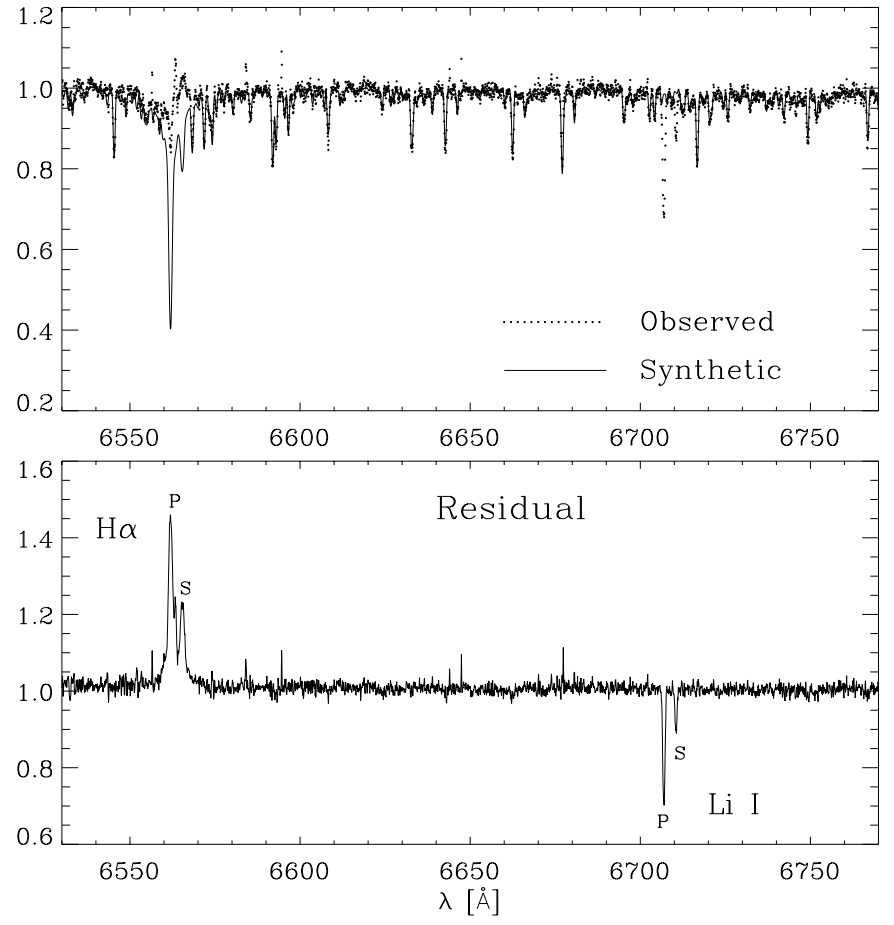

Fig. 3. An example of application of the spectral subtraction procedure to the star RX J0541-0324: in the upper panel, the observed spectrum is drawn as a dotted line, whereas the synthetic one, obtained combining the spectra of a G8 and a K3 standard star, as a solid line; the residual spectrum is shown in the lower panel, and the $\mathrm{H} \alpha$ emission and lithium absorption lines of the primary and secondary components are marked with $\mathrm{P}$ and $\mathrm{S}$, respectively.

trum from that of the binary. The synthetic spectrum was built up using two stars of appropriate spectral type, for the primary and secondary component respectively, acquired with the same instrumental set-up. This procedure offers the advantage of eliminating any possible contamination of the lithium line from blending with nearby iron lines (Lee et al. 1994).

Independent measurements for each star have been obtained from at least three different FOCES spectra of higher $S / N$ ratio and where the components are seen best separated and we estimate a mean error of $15 \mathrm{~m} \AA$ on the observed equivalent width. Li $16708 \AA$ equivalent widths, $W(\mathrm{Li})$, have then been corrected by the corresponding weighting factors derived from the synthetic spectrum fit, to account for the actual contribution of each star to the total continuum flux. The average, corrected lithium equivalent widths are given in Table 5 . The errors on $W(\mathrm{Li})$ have been estimated determining the $S / N$ ratio in two windows on the right and left-hand side of the $\mathrm{Li}$ line in the residual spectrum and taking into account the errors on the weighting factors.

The lithium equivalent width versus effective temperature for the binary components is shown in Fig. 4. For comparison, the upper envelope for young open cluster adopted by Martín \& Magazzù (1999) is drawn as a thick line, whereas the locii of PMS stars, distinguished as 


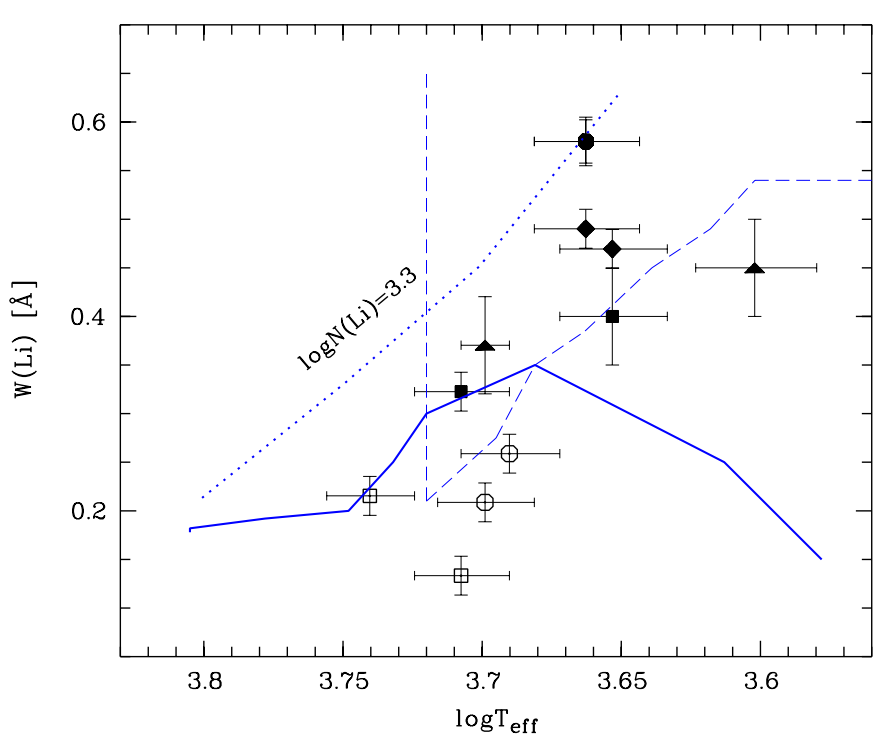

Fig. 4. Lithium equivalent widths versus effective temperature for the individual components of the six binaries with spectroscopic orbits solved in this paper. For comparison, the upper envelope for young (ZAMS) open clusters is drawn as a thick solid line and the location of PMS stars, as TTS and PTTS, according to the classification scheme proposed by Martín (1998), is indicated by the long-dashed lines. Symbols as in Fig. 5. Note that the two equal mass components of RX J0530.7-0434 coincide in position

T Tauri (TTS) and post-T Tauri stars (PTTS), according to the criterion introduced by Martín (1998), are delimited by the long-dashed lines.

Lithium abundance of each component is then derived by using the theoretical curves of growth based on NLTE atmospheric models by Pavlenko \& Magazzù (1996), assuming $\log g$ of 4.5. The latter assumption is the most conservative one as, for a given effective temperature, this value of $\log g$ yields a lower lithium abundance, as also discussed in Paper II. The lithium abundances derived in this way are reported in Table 5. The main source of error on the derived $\log N(\mathrm{Li})$ is the uncertainty on the spectral type, which translates in an uncertainty on the effective temperature of about $200 \mathrm{~K}$. This yields a mean error of about 0.3 dex on the derived abundance. Lithium abundance versus effective temperature is shown in Fig. 5, for individual binary components and, as a reference, for stars in the young ( $\approx 30$ Myr old) open cluster IC 2602 from Randich et al. (1997).

\subsection{Spatial location and kinematics}

The other important criterion for assessing that the stars are physically associated with the Orion SFR is based on kinematics. Systemic radial velocities can be compared with the radial velocities of known Orion members, as well as of the gas in the region provided by $\mathrm{CO}$ observations available from the literature (Maddalena et al. 1986).

Figure 6 shows the spatial location of the six binaries in the general direction of Orion, compared to the distribution of the gas, provided by the $\mathrm{CO}$ contour map (thick

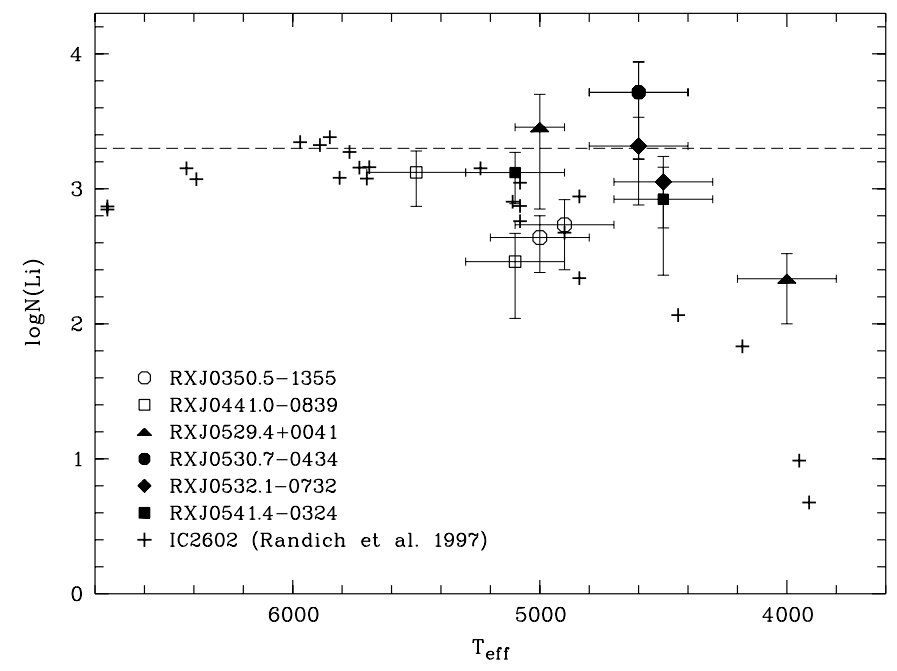

Fig. 5. Lithium abundance versus effective temperature for the individual components of the binaries in our sample. Stars of the young open cluster IC 2602 (small crosses) are also shown for comparison.

line), and with that of dust grains, provided by the $100 \mathrm{mi}-$ cron IRAS contours. It is indeed striking the fact that all the systems, with the only exception of RX J0350.5-1355, are seen projected against regions of enhanced $\mathrm{CO}$ and/or infrared dust-emission and that their systemic radial velocities are fairly consistent with the radial velocity of the gas and of other PMS stars in the Orion molecular cloud (OMC). It is also remarkable that the four binaries with the largest lithium content seem to be related, both spatially and kinematically, to the Orion SFR. The other two systems, which are seen projected more far apart from the SFR, might be somewhat older. In particular, RX J0350.51355 appears clearly unrelated to the SFR, and it is probably a more evolved system.

\subsection{Position on the HR diagram}

As the dynamically determined mass products, $M_{1,2} \sin ^{3} i$, provide lower limits for the masses of the binary components, one can use these values, and their ratio, to check whether they are compatible with the masses inferred from the theoretical evolutionary tracks. Most interesting to this respect are the binary systems with a mass ratio different from unity because they allow to set constraints on both theoretical evolutionary tracks and isochrones.

In order to examine the position of the binary components on the Hertzsprung-Russell diagram (HRD) relatively to different sets of theoretical PMS evolutionary tracks, luminosity ratios and spectral types of the individual components have been evaluated as described in Sect. 3.3. Except for the case of the eclipsing system RX J0529.4+0041, for which a distance of 360 pc has been derived (Covino et al. 2000), for the other three systems with Right Ascension larger than 5 hours, which appear spatially and kinematically related to the OMC, the general distance of $460 \mathrm{pc}$ to the Orion complex has 


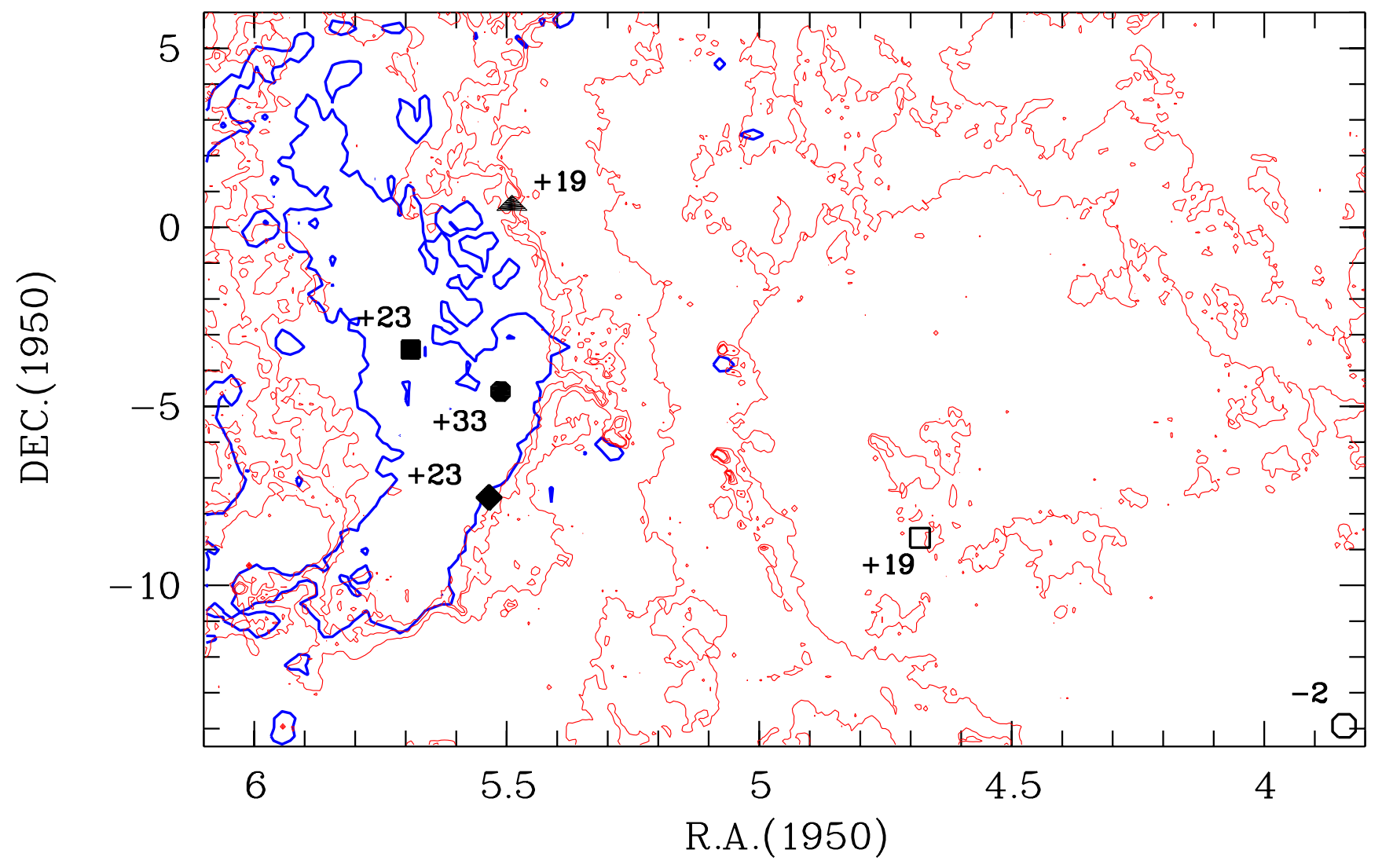

Fig. 6. Spatial distribution of the six binaries with resolved spectroscopic orbit (symbols as in Fig. 5). Each star is marked with the corresponding systemic radial velocity. Also shown are the CO emission contours from Maddalena et al. (1986) (thick line) and the $100 \mu \mathrm{m}$ IRAS contours corresponding to $5 \div 30 \mathrm{mJy} \mathrm{sr}^{-1}$ (thin line).

been adopted (Genzel \& Stutski 1989). No distance determination is available for the remaining two systems, RX J0350.5-1355 and RX J0441.0-0839, and, on the other hand, the assumption of the Orion distance does not appear justified as they are seen projected too far away from the OMC and their systemic radial velocities are inconsistent with the kinematics of the gas. For that reason, no attempt is made to locate the components of these two systems on the HRD.

In the case of RX J0530.7-0434, RX J0532.1-0732 and RX J0541.4-0324, total luminosities have been derived as described in Alcalá et al. (1997), and the luminosities of the individual components are estimated adopting the luminosity ratios given in Table 5 . Only in the case of the eclipsing system, RX J0529.4+0041, the luminosity of the components can be obtained independently from the distance, as described in Covino et al. (2000). Figure 7 shows the position on the HRD of the components of the 4 binaries in Orion, for which a distance estimate as well as absolute $U B V R I, J H K$ photometry are available (Paper I). The comparison with the different sets of theoretical tracks is discussed in Sect. 5 .

\section{Evolutionary status of individual systems}

\subsection{RX J0350.5-1355}

RX J0350.5-1355 was already known from previous X-ray observations of the Einstein satellite Extended Medium
Sensitivity Survey and reported as a spectroscopic binary star of spectral type K0IV, with designation 1E 0348.21404, by Fleming et al. (1989). From the orbital solution we derive that RX J0350.5-1355 has a circular orbit and an orbital period of about 9.3 days.

It is particularly important to establish unambiguously the evolutionary status of this system because, if the PMS nature of RX J0350.5-1355 should be confirmed, it would represent the longest period system with a circular orbit presently known. As no distance determination is available for this object, it is not posssible to locate the binary components on the HRD. However, based on its relatively low lithium content, it seems rather unlikely that RX J0350.51355 is a PMS star. Moreover, the object is located quite away from the bulk of the Orion SFR and its systemic radial velocity also appears inconsistent with the kinematics of the gas in that region. Based on this, we cannot exclude the possibility that RX J0350.5-1355 is a more evolved system and, given the late spectral types and the relatively high masses inferred for the components, it might be an active binary belonging to the RS CVn class (Pallavicini et al. 1992; Strassmeier et al. 1993).

Light-curve modulation with a periodicity very close to the orbital period is also inferred from the available photometry (Covino et al. 2001), suggesting that both tidal circularization of the orbit and synchronization of rotational and orbital periods have been achieved in this 

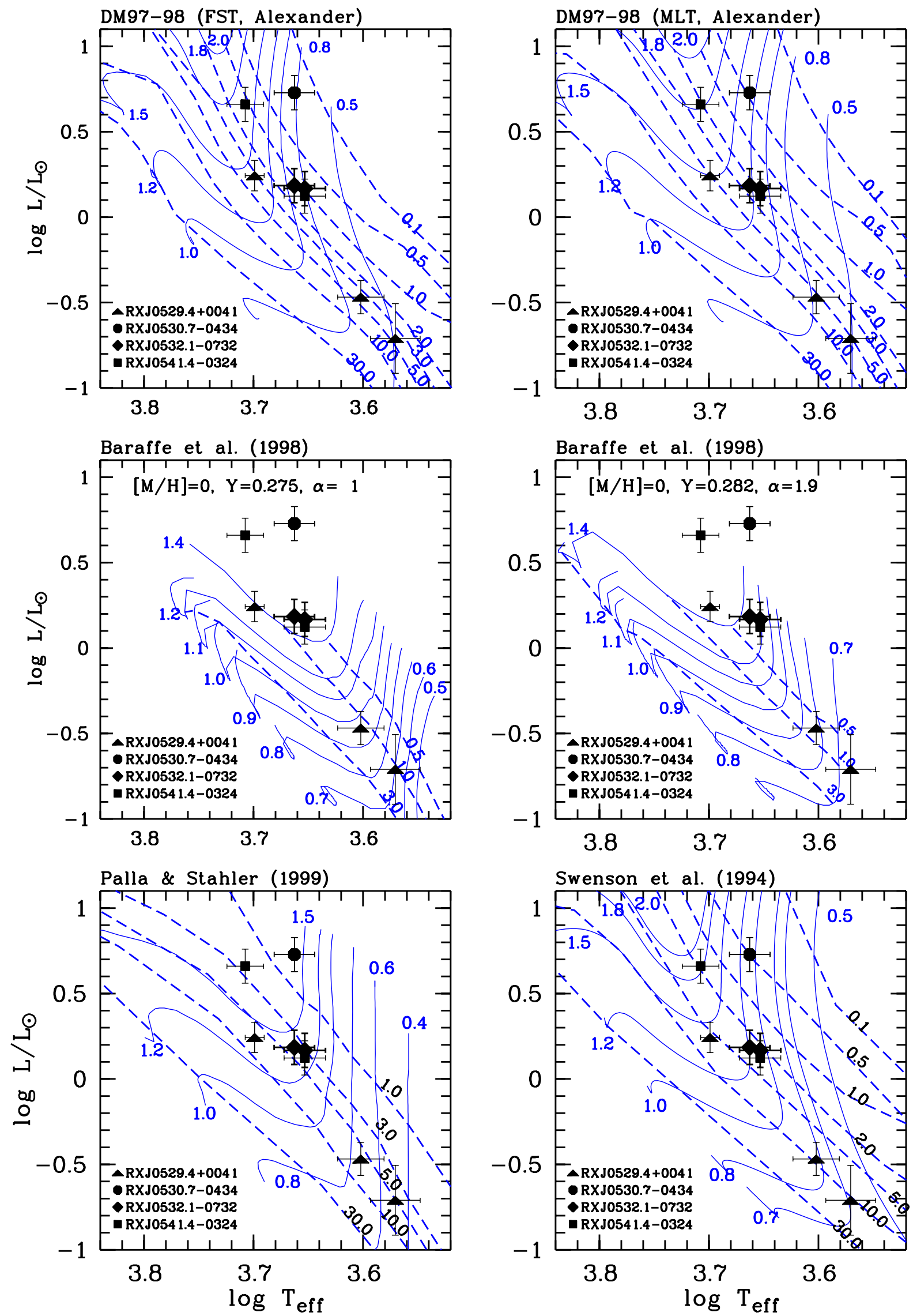

Fig. 7. Hertzsprung-Russel diagram for the 4 bona fide PMS spectroscopic binaries in our sample. Comparison is made with four different sets of PMS evolutionary tracks. Isochrones are labelled in units of 10 Myr. The two equal mass components of RX J0530.7-0434 coincide at the same position. 
system. If this is the case, then the larger $v \sin i$ $\left(19 \mathrm{~km} \mathrm{~s}^{-1}\right)$ of the primary with respect to the secondary component (about $7 \mathrm{~km} \mathrm{~s}^{-1}$ ) must reflect a proportionally larger radius of the primary component. Furthermore, the larger scatter in the radial velocities for the primary is probably due in part to the faster rotation of that component, which makes the spectral lines shallower, and probably also to its strong magnetic activity. In fact, intrinsic phenomena such as stellar spots, responsible for the observed 0.2 photometric wave, can also give rise to spectral line distortions producing extra "jitter" in the radial velocities.

\section{2. $R X$ J0441.0-0839}

This system has an orbital period of 13.6 days and an orbital eccentricity of about 0.2. Similarly to RX J0350.51355 it is also located off the galactic plane and relatively far from the bulk of the Orion SFR, but still within the boundaries of the Gould belt (see Fig. 6), with a systemic radial velocity of about $19 \mathrm{~km} \mathrm{~s}^{-1}$, which is consistent with the kinematics of that region.

RX J0441.0-0839 is a close visual pair with an angular separation of about 1.5 arcsec. The visual companion, also detected spectroscopically, appears to be brighter than the double-lined binary at visual wavelengths (estimated $\left.L_{3} / L_{1}=2.0 \pm 0.1\right)$. Therefore, the orbital elements presented in Table 4 actually refer to the visual secondary. We find that the third star rotates faster than the other two components $\left(v_{3} \sin i \simeq 24 \mathrm{~km} \mathrm{~s}^{-1}\right)$. Our radial velocity measurements for the third star show no significant variation, and the average is within $1 \mathrm{~km} \mathrm{~s}^{-1}$ of the centerof-mass velocity of the spectroscopic binary, strongly suggesting physical association. Thus, this is very likely a hierarchical triple system.

In all of the three spectroscopically visible components of RX J0441.0-0839 the Li I $\lambda 6708 \AA$ resonance line appears slightly weaker than the adjacent Ca I $\lambda 6718 \AA$ line and the lithium equivalent widths, after accounting for the dilution effect arising from the superposition of the three different components, fall below the upper boundary for zero-age main-sequence (ZAMS) stars (see Fig. 4). The spectral types of the components and the masses inferred from the orbital solution seem to be more consistent with those of main sequence stars. Therefore, we conclude that RX J0441.0-0839 is probably not a PMS system but it could have already reached the ZAMS.

\section{3. $R X J 0529.4+0041$}

With an orbital period just over 3 days, RX J0529.4+0041 is the shortest period binary in our sample. From the spectroscopic orbital solution, the primary and secondary components result to have minimum masses of about 1.3 and $0.9 M_{\odot}$, respectively, yielding a mass ratio of about 0.7 . Furthermore, RX J0529.4+0041 has been recently found to be an eclipsing binary, thus absolute masses as well as effective temperatures and radii could be derived for the two components (Covino et al. 2000).

The spatial location of RX J0529.4+0041, close to the north-east side of the Orion cloud B, and its systemic radial velocity of about $20 \mathrm{~km} \mathrm{~s}^{-1}$ strongly support the membership to the SFR. Examination of the lithium content of the components provides a further indication that the system is definitely very young with a quite high lithium abundance (see Fig. 5), comparable to that observed in classical T Tauri stars. Based on this observational evidence, Covino et al. (2000) conclude that RX J0529.4 +0041 is a bona-fide PMS binary, with an estimated age not larger than 10 Myr. Interestingly, this system is also very likely a hierarchical triple, as the presence of a third fainter component is revealed both spectroscopically, at a radial velocity close to the systemic value (see Fig. 2), and as a visual companion, at an angular separation of about 1.3 arcsec. We estimate the projected rotational velocity of the third star to be approximately $9 \mathrm{~km} \mathrm{~s}^{-1}$. Adopting for the third component the same distance of 360 pc inferred for the eclipsing binary, Covino et al. (2000) estimated the luminosity of this visual companion from near-infrared photometry and it is thus possible to locate it on the HRD. Remarkably, the three components of RX J0529.4+0041 appear to lie quite close to the same isochrone, indicating an age of the order of $10 \mathrm{Myr}$, consistent with the age of the Ori OB1a association on which this system appears projected.

\section{4. $R X J 0530.7-0434$}

It is immediately clear from the appearance of the composite spectrum that the mass ratio of the components is nearly 1 , as the two components are very similar in both spectral type and luminosity, therefore, they share the same position on the HR diagram. The spectral type is estimated to be between $\mathrm{K} 2$ and $\mathrm{K} 3$ for both the primary and the secondary component and the products $M_{1,2} \sin ^{3} i$, are about $1.5 M_{\odot}$ for each star.

RX J0530.7-0434 was initially regarded by us as a good candidate to be an eclipsing system because of the large minimum masses of the components. However, although photometric variations (apparently related to the orbital phase) have been observed, no evidence for the occurence of eclipses has been found in this system. A periodogram analysis of available photometric data revealed that the variations have a possible periodicity of about 13.5 days (Covino et al. 2001), which corresponds to nearly $1 / 3$ of the orbital period. On the assumption that this is the true rotation period, and using the photometric radius derived from the absolute luminosity and effective temperature in Table 5, the predicted equatorial rotation rate is essentially identical to the $v \sin i$ value that we measure (see Table 5). In addition to suggesting that the rotational axis is nearly perpendicular to the line of sight, this supports the hypothesis that the rotation period is indeed 13.5 days, and it would also imply that rotation is not synchronous 
with the orbital motion. The latter is actually expected, at least qualitatively, on the basis of the long orbital period of the system and its significant eccentricity. However, the fact that the photometry corresponds to the combined light of the two components complicates the argument, although in principle the two stars could very well have similar rotation periods since they are essentially identical in all their other properties (mass, radius, effective temperature), and have presumably also had similar angular momentum histories.

The combined apparent magnitude of the system is 11.5 in $\mathrm{V}$ (corrected for interstellar extinction), yielding a total luminosity of about $11 L_{\odot}$, at the distance of $460 \mathrm{pc}$, and a luminosity of about $5.3 L_{\odot}$ for the individual components. Figure 7 shows the position of the components of RX J0530.7-0434 in the HRD after correcting for the duplicity factor, and assuming that the system is located in the Orion star formation region at the distance of $460 \mathrm{pc}$.

All the observational evidences considered here (e.g. spatial location, kinematics, lithium abundance, as well as position on the HRD) strongly indicate that RX J0530.70434 is a bona-fide PMS system and that it is also probably the youngest of the systems in our sample, with an estimated age of a few $10^{6}$ years.

From the comparison with the different sets of theoretical PMS evolutionary tracks considered in Fig. 7, it appears that, although different theoretical masses are inferred from each set, these are consistent with the dynamical determinations in all cases except the D'Antona \& Mazzitelli (1997) set computed with the Canuto \& Mazzitelli's (1991) full spectrum of turbulence (FST) convection treatment.

\section{5. $R X J 0532.1-0732$}

This spectroscopic binary with an orbital period of about 47 days is the longest period system in the present sample. The orbital eccentricity is rather high $(e=0.47)$, while the mass ratio, $q=0.95$, is not too different from unity. As in two other of our systems, we have detected in our spectra the presence of a faint third component with a brightness in the visual band of $L_{3} / L_{1}=0.18 \pm 0.03$, and we have been able to measure its radial velocity. The average value is once again close to the systemic velocity of the close pair, with no significant variation, suggesting physical association (hierarchical triple). The third component is relatively sharp-lined $\left(v \sin i \sim 10 \mathrm{~km} \mathrm{~s}^{-1}\right)$ compared to the other two stars.

In line with their similar masses, the spectral types and luminosities of the primary and secondary stars do not differ much, and their position in the HR diagram is near the $3 \mathrm{Myr}$ isochrone, between the 1.4 and $1.2 \mathrm{M}_{\odot}$ tracks. These values are consistent with our dynamical determination of the minimum masses for the stars.

RX J0532.1-0732 is located on the eastern edge of the Orion cloud $\mathrm{A}$ and the radial velocity of its center of mass is about $23 \mathrm{~km} \mathrm{~s}^{-1}$, in fairly good agreement with the velocity of the gas in that region. The age derived from the position on the HR diagram, between the 3 and $5 \mathrm{Myr}$ isochrones, is also consistent with the age determinations for other stars in the same region. Finally, the extreme youth of the system is also shown by the rather high lithium content of the components, $\log N(\mathrm{Li})$ of 3.2 and 3.1, respectively, in the usual scale. Therefore, we can firmly conclude that the components of RX J0532.1-0732 are bona fide PMS stars.

\section{6. $R X J 0541.4-0324$}

This system has a circular orbit and an orbital period of nearly 5 days. The solution of the spectroscopic orbit yields minimum masses for the components of 1.3 and $0.9 M_{\odot}$, respectively, and a mass ratio of about 0.67 . However, the comparison of the position of the two components in the HR diagram yields, for all sets of tracks, theoretical masses which are well above the dynamical minimum masses. Thus, it seems very unlikely that this short-period system can be an eclipsing binary.

Both the relatively high lithium content and the position on the HRD of the components indicate that RX J0541.4-0324 is a PMS binary with estimated age between 5 and $10 \mathrm{Myr}$. Also quite noticeable appears the fact that the object is seen projected very close to the Orion Nebula. However, we notice that, according to the $T_{\text {eff }}$ boundary proposed by Martín (1998) in the Li equivalent width versus effective temperature diagram, only the secondary component would be classified as a PMS star, thus showing the limitation of a purely lithium-based classification scheme.

\section{Comparison with PMS evolutionary tracks}

In Fig. 7 the position on the HRD of individual components of the four bona fide PMS systems is compared with some of the most commonly used sets of PMS evolutionary tracks and isochrones available in the literature, namely: i) D'Antona \& Mazzitelli (1997, DM97) set with Alexander opacities and full spectrum of turbulence (FST) convection treatment by Canuto \& Mazzitelli (1991); ii) Baraffe et al. (1998) for low-mass solar metallicity stars; iii) Palla \& Stahler (1999); iv) Swenson et al. (1994). It was already well known that DM97 tracks with the FST convection treatment tend to predict lower masses and younger ages as compared to other PMS evolutionary tracks. For the components of the eclipsing system, RX J0529.4+0041, absolute masses could be determined, thus allowing for a direct comparison with the prediction from the theoretical models. For the other three PMS systems, due to the indetermination on the inclination of the orbital plane, only minimum values of the dynamical mass are obtained from the spectroscopic orbit solution. Yet, these minimum values for the masses set an important lower limit for the theoretical masses and, combined with the information on the mass ratios, provide a useful constraint on the models. Figure 8 shows the comparison of 

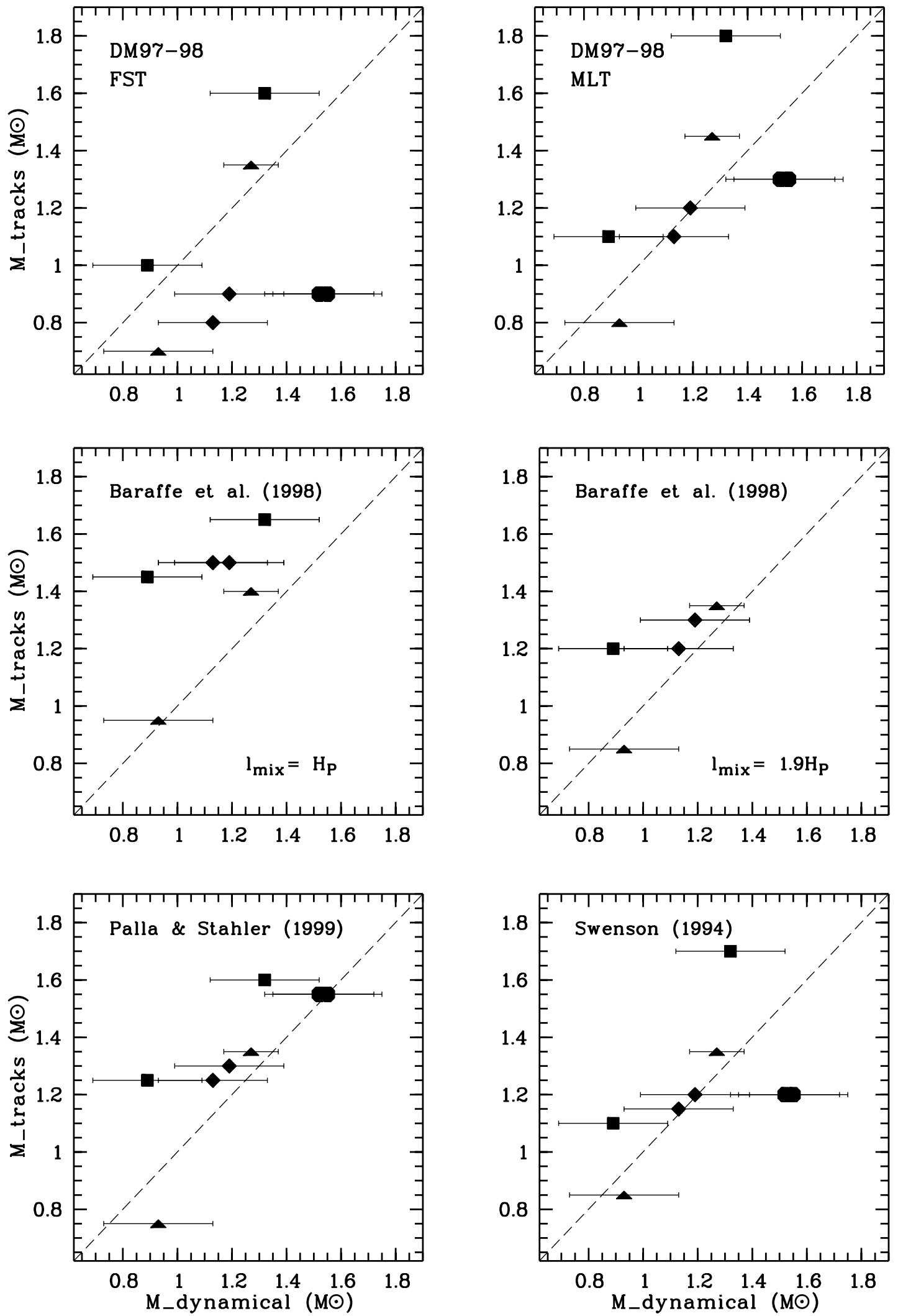

Fig. 8. Theoretical masses versus dynamical mass determinations of the PMS binary components, for the sets of tracks considered in Fig. 7 (symbols are also the same). In all cases, except for the eclipsing system RX J0529.4+0041 for which the orbital inclination has been determined, the value reported in abscissa represents the lower limit for the dynamical mass, $M \sin ^{3} i$. Thus, the $45^{\circ}$ dashed line marks the lower boundary for the allowed theoretical mass. 
the theoretical mass against the minimum dynamical mass (for the two eclipsing components of RX J0529.4+0041, the true dynamical masses are used). Points falling below the 45 degrees dashed line in this diagram reveal inconsistences in the models.

It is apparent from this comparison that, for masses below about $1 M_{\odot}$, DM97+FST tracks are inconsistent with the dynamical values, whereas the remaining three sets of tracks predict masses which seem to be in better agreement, within the errors, with the dynamical mass determinations. In all cases, the comparison with PMS theoretical isochrones indicates that the two components in each of the investigated systems appear to be coeval within observational uncertainties.

A detailed discussion of the possible causes of inconsistences between model predictions and observations falls beyond the scope of the present paper, however it is worthwhile to remark that the convection treatment still remains a very crucial point in the modeling of low-mass PMS stars (D'Antona 2000). On the other hand, the very recent calculations by D'Antona et al. (2000) have also clearly shown how sensitively modifications to the atmospheric thermal structure due to the magnetic field can affect the location in effective temperature of the PMS tracks on the HRD.

\section{Summary and conclusions}

We have presented spectroscopic orbits for six spectroscopic binaries previously identified in the general direction of the Orion star forming region on the basis of the ROSAT X-ray all-sky survey and optical follow-up observations.

Using the information derived from solution of the spectroscopic orbits combined with ancillary criteria based on the lithium content, spatial location, and kinematics, we are able to disentangle in an unambiguous way the bona-fide PMS systems from more evolved ones. This allows us to fully confirm the PMS nature for 4 of the 6 investigated systems, whereas the remaining two, RX J0350.5-1355 and RX J0441.0-0839, are recognized to be more evolved. The former might be a post-main sequence binary, of RS CVn type, while the latter system is presumably composed of two zero-age main sequence stars.

The four PMS SB2 systems presented here contribute to enlarge the statistics of known short-period PMS binaries. In fact, according to the latest compilation of known PMS spectroscopic binaries by Melo et al. (2001), already including these 4 new systems, the total number of spectroscopic binaries with solved orbits amounts to nearly 40 , whereas the number of double-lined spectroscopic binaries with orbital period shorter than 100 days rises now to 23 .

Estimated lithium abundances for individual components also turn out to be fairly consistent with the expected lithium depletion in low-mass PMS stars of similar age.
The comparison with PMS theoretical isochrones on the HRD provides indication that, within observational uncertainties, the two components in a binary system are found to be coeval in all the considered cases. Furthermore, positioning on the HR diagram of individual binary components and comparison between the dynamical minimum masses and the theoretical masses inferred from different sets of most commonly used PMS evolutionary tracks provide a valuable constraint on the theoretical models.

Finally, an interesting finding in our admittedly small sample of stars is the strikingly high proportion of hierarchical triple systems: two among the four binaries considered to be bona-fide PMS have been found to have an additional component very likely to be physically associated. This is consistent with the general evidence for a high multiplicity among young stars (see, e.g., Leinert et al. 1993; Simon et al. 1995; Ghez et al. 1997).

Acknowledgements. We wish to thank Franca D'Antona, Francesco Palla, Sergei Lamzin and Michael Sterzik for stimulating discussions. We are grateful to Michel Mayor for giving strong support to this programme and to Ralph Neuhäuser for triggering the contact with CfA in the initial stages of this project. We thank the technical staff of Calar Alto Observatory for their support during the observations; special thanks go to Michael Pfeiffer, whose premature loss deeply saddened all of us, for his great work in making FOCES run and for his very nice friendship. We are grateful to P. Berlind, M. Calkins, J. Caruso, D. W. Latham, A. Milone, and R. P. Stefanik for obtaining many of the spectroscopic observations at CfA, and to Bob Davis for maintaining the CfA database of radial velocities. CHFM acknowledges grants from CPNq (proc. 200614/96-7) and Fonds Marc Birkigt as well as the Capodimonte Observatory (OAC) for hospitality and support during two stays in Naples. This research has made use of the Simbad database, operated at CDS, Strasbourg, France. This work was financed in part by the Italian Ministero dell'Università e della Ricerca Scientifica $e$ Tecnologica (cofin98). Finally, we wish to thank the referee, Dr. Didier Queloz, for his helpful comments.

\section{References}

Alcalá, J. M., Terranegra, L., Wichmann, R., Chavarria, C., \& Krautter, J. 1996, A\&AS, 119, 7 (Paper I)

Alcalá, J. M., Krautter, J., Covino, E., et al. 1997, A\&A, 319, 184

Alcalá, J. M., Covino, E., Torres, G., et al. 2000, A\&A, 353, 186 (Paper II)

Baraffe, I., Chabrier, G., Allard, F., \& Hauschildt, P. H. 1998, A\&A, 337, 403

Baranne, A., Queloz, D., Mayor, M., et al. 1996, A\&AS, 119, 373

Canuto, V. M, \& Mazzitelli, I. 1991, ApJ, 370, 295

Covino, E., Alcalá, J. M., Allain, S., et al. 1997, A\&A 328, 187

Covino, E., Catalano, S., Frasca, A., et al. 2001, Proc. of the XI Cambridge workshop on Cool Stars, Stellar Systems and the Sun: Challenges for the New Millennium, ed. R. J. Garcia Lopez, R. Rebolo, \& M. R. Zapatero Osorio, ASP Conf. Ser., 223, CD-503

Covino, E., Catalano, S., Frasca, A., et al. 2000, A\&A, 361, L49 
D'Antona, F. 2000, in 33rd ESLAB Symp., Star Formation from the Small to the Large Scale, ESA SP-445, in press

D'Antona, F., \& Mazzitelli, I. 1997, in Cool Stars in Clusters and Associacions: magnetic activity and age indicators, ed. G. Micela, R. Pallvicini, \& S. Sciortino, Mem. Soc. Astron. It., 68, 807

D’Antona, F., Ventura, P., \& Mazzitelli, I. 2000, ApJ, 543, L77

de Jager, C., \& Nieuwenhuijzen, H. 1987, A\&A, 177, 217

Fleming, T. A., Gioia, I. M., \& Maccacaro, T. 1989, ApJ, 340, 1011

Genzel, R., \& Stutski, J. 1989, ARA\&A, 27, 41

Ghez, A. M., White, R. J., \& Simon, M. 1997, ApJ, 490, 353

Gray, D. F. 1976, The observation and analysis of stellar photospheres (Wily \& Sons Inc.), 394

Kurucz, R. L. 1992a, in The Stellar Population of Galaxies, IAU Symp. 149, ed. B. Barbuy, \& A. Renzini (Reidel, Dordrecht), 225

Kurucz, R. L. 1992b, Rev. Mex. Astron. Astrofís, 23, 187

Kurtz, M. J., \& Mink, D. J. 1998, PASP, 110, 934

Latham, D. W., Nordström, B., Andersen, J., et al. 1996, A\&A, 314,864

Lee, C. W., Martín, E. L., \& Mathieu, R. D. 1994, AJ, 108, 1445

Leinert, C., Zinnecker, H., Weitzel, N., et al. 1993, A\&A, 278, 129

Maddalena, R. J., Morris, M., Moscowitz, J., \& Taddeus, P. 1986, ApJ, 303, 375

Martín, E. L. 1998, AJ, 115, 351

Martín, E. L., \& Magazzù, A. 1999, A\&A, 342, 173

Mathieu, R. D., R. D., Ghez, A. M., Jensen, E. L. N., \& Simon, M. 1999, Protostars \& Planets IV (Tucson Univ. of Arizona Press), 703

Mayor, M., \& Mermilliod, J.-C. 1984, in Observational tests of the Stellar Evolution Theory, ed. A. Maeder, \& A. Renzini (Reidel, Dordrecht), IAU Symp. 105, 411
Melo, C., Covino, E., Alcalá, J. M., \& Torres, G. 2001, A\&A, submitted

Neuhäuser, R., Sterzik, M. F., Schmitt, J. H. M. M., Wichmann, R., \& Krautter, J. 1995, A\&A, 297, 391

Palla, F., \& Stahler, S. W. 1999, ApJ, 525, 772

Pallavicini, R., Randich, S., \& Giampapa, M. S. 1992, A\&A 253, 185

Pavlenko, Y. P., \& Magazzù, A. 1996, A\&A, 311, 961

Pfeiffer, M. J., Frank, C., Baumüller, D., Fuhrmann, K., \& Gehren, T. 1998, A\&AS, 130, 381

Press, W. H., Teukolsky, S. A., Vetterling, W. T., \& Flannery, B. P. 1992, Numerical Recipes in Fortran. The Art of Scientific Computing, 2nd Ed. (Cambridge: Cambridge Univ. Press), 650

Queloz, D. 1995, in IAU Symposium 167, New Developments in Array Technology and Applications, ed. A. G. Davis Philip (Dordrecht: Kluwer), 221

Queloz, D., Allain, S., Mermilliod, J.-C., Bouvier, J., \& Mayor, M. 1998, A\&A, 335, 183

Queloz, D., Mayor M., Naef, D., et al. 2000, in VLT Opening Symposium, From extrasolar planets to brown dwarfs, ESO Astrophys. Symp., ed. J. Bergeron, \& A. Renzini (Springer Verlag, Heidelberg), 548

Randich, S., Aharpour, N., Pallavicini, R., Prosser, C. F., \& Stauffer, J. R. 1997, A\&A, 323, 86

Simon, M., Ghez, A. M., Leinert, Ch., et al. 1995, ApJ, 443, 625

Strassmeier, K. G., Hall, D. S., Fekel, F. C., \& Scheck, M. 1993, A\&AS, 100, 173

Swenson, F. J., Faulkner, J., Rogers, F. J., \& Iglesias, C. A. 1994, ApJ, 425, 286

Zucker, S., \& Mazeh, T. 1994, ApJ, 420, 806

Zucker, S., Torres, G., \& Mazeh, T. 1995, ApJ, 452, 863 